\title{
Evo-Devo of Infantile and Childhood Growth
}

\author{
ZE'EV HOCHBERG AND KERSTIN ALBERTSSON-WIKLAND \\ Meyer Children's Hospital [Z.H.], Faculty of Medicine, Technion - Israel Institute of Technology, Haifa, IL-31096, Israel; Pediatric \\ Growth Research Center, Department of Pediatrics [K.A.-W.], The Institute of Clinical Science, Sahlgrenska Academy at University \\ of Gothenburg, Gothenburg S-41685, Sweden
}

\begin{abstract}
Human size is a tradeoff between the evolutionary advantages and disadvantages of being small or big. We now propose that adult size is determined to an important extent during transition from infancy to childhood. This transition is marked by a growth spurt. A delay in the transition has a lifelong impact on stature and is responsible for $44 \%$ of children with short stature in developed countries and many more in developing countries. Here, we present the data and theory of an evolutionary adaptive strategy of plasticity in the timing of transition from infancy into childhood to match the prevailing energy supply. We propose that humans have evolved to withstand energy crises by decreasing their body size, and that evolutionary short-term adaptations to energy crises trigger a predictive adaptive response that modify the transition into childhood, culminating in short stature. (Pediatr Res 64: 2-7, 2008)
\end{abstract}

$\mathrm{E}^{\mathrm{s}}$ volutionary developmental biology (evo-devo) addresses the issues of how developmental systems have evolved and probes the consequences of these historically established systems for organismal evolution (1). Research in evo-devo has formed around comparative embryology and morphology, evolutionary developmental genetics, and experimental epigenetics. Here, we take evo-devo into the realm of clinical medicine and child growth.

Humans are born immature and helpless, have a relatively short period of infancy, and are the only species that have a childhood-a biologically and behaviorally distinct and relatively stable interval between infancy and the juvenile period that follows (2). The transition from infancy to childhood (IC) is marked by a growth transition. A delay in this growth transition, which we refer to as delayed infancy childhood transition (DICT), has a lifelong impact on stature and is responsible for as many as $44 \%$ of children with a normal birth weight and no endocrine disease, who are referred to pediatric endocrine clinics as suffering from idiopathic short stature (ISS).

Here, we present the data and theory that there is an evolutionary adaptive strategy of plasticity in the timing of the transition from infancy into childhood to match environmental cues and energy supply. We propose that humans evolved to withstand energy crises by decreasing their body size, and that

Received January 29, 2008; accepted March 21, 2008

Correspondence: Ze'ev Hochberg, M.D., Ph.D., Meyer Children's Hospital, POB 9602, Haifa 31096, Israel; e-mail: z_hochberg@rambam.health.gov.il

K.A.W. is supported by a Swedish Research Council grant 7905. evolutionary short-term adaptations to energy crises use epigenetic mechanisms that modify the transition into childhood, culminating in short stature.

\section{STAGES OF LIFE HISTORY}

The approach to human life history adopted here was developed by Bogin (3). It is a biologic and behavioral model of human development, based on a consideration of comparative mammalian biology and primate evolutionary history. Life history is the product of strategies an organism uses to allocate its energy toward growth, maintenance, reproduction, raising offspring to independence, and avoiding death (4). For a mammal, it is the strategy of when to be born, when to be weaned, when to be independent for self-protection and provision, when and how often to reproduce, and when to die. During human evolution, childhood and adolescence have been added as new life stages as compared with apes (4). Thus, Homo sapiens have four prolonged and pronounced preadult life history stages: infancy, which extends for 30-36 mo; childhood, which extends for 4-6 y between infancy and adrenarche; a juvenile stage, which lasts for $2-4$ y (2); and adolescence, which lasts for 3-5 y.

Anthropologists attempt to explain how the human species has survived in the face of its unique life history: a helpless neonatal period with a short duration of breastfeeding; an extended childhood when the offspring are still dependent for protection and food provision on parental and community assistance; a juvenile period when they mostly provide for and are able to look after themselves, and when competition with adults for food and space is possible only because the offspring are sexually immature; an energy-costly adolescent growth spurt; delayed reproduction; menopause; and a uniquely long postmenopausal life.

Evolution of this life history has involved the gradual cumulative selection and development of traits over millions of years. As a major consumer of energy, growth plays a foremost role, and the ability to vary growth patterns and the rate of growth during particular life stages - to shorten or prolong life stages and

Abbreviations: DICT, delayed infancy to childhood transition; GHD, growth hormone deficiency; IC, infancy-childhood; ICP, infancy-childhoodpuberty; ISS, idiopathic short stature; SDS, standard deviation score; SGA, small for gestational age 
even to add or delete life stages—provides a compelling strategy. The latter is exemplified by the addition of a childhood stage by Homo habilis 1.9 million years ago.

\section{INFANCY AND CHILDHOOD}

Bipedalism of the hominids required realignment of the spine and legs and a narrowing of the pelvis, whereas a more developed and neurologically more advanced brain required a large skull. This was described as an "obstetrical dilemma" by Washburn in 1960 (5), who proposed that, as an adaptation to this unfavorable ratio between a smaller maternal birth canal and a larger fetal skull, head and brain growth (and motor development) have been deferred into the postnatal period. Brain growth tapers off after birth in other primates, but continues in a rapid, fetal-like trajectory in humans for the first year of postnatal life.

The life stage of infancy has been defined by weaning in traditional societies at about $30-36$ mo of age. If we consider the lifespan of humans, the period of infancy is markedly shorter than, for example, the 60-mo infancy of the chimpanzee, which lives for $40 \mathrm{y}$ in the wild. In humans and other mammals, infancy is characterized by rapid and decelerating growth, deciduous dentition, and feeding by maternal lactation. Although the duration of infancy is shorter in humans than in other mammals, its quality is higher. Greater physical support by the human infant produces more intense social stimulation, and during this period, the brain grows rapidly. Unique to humans is a long interval of $3 \mathrm{y}$ between the end of infancy and the eruption of the first permanent molar tooth, and it is within this interval that the childhood period developed.

Piaget defined infancy as the sensorimotor period-from birth to about age 2 (Table 1) (6). During this stage, the child learns about himself and his environment through motor and reflex actions. Thought derives from sensation and movement. The child learns that he is separate from his environment, and that aspects of his environment-his parents or favorite toy-continue to exist even though they may be outside the reach of his senses.

The childhood stage is peculiar to humans, and has been defined by stabilization of the growth rate, immature dentition,

Table 1. Piaget's stages of cognitive development

\begin{tabular}{|c|c|}
\hline Approximate age & $\begin{array}{l}\text { Developmental stage and } \\
\text { characteristic behavior }\end{array}$ \\
\hline $0-24 \mathrm{mo}$ & $\begin{array}{l}\text { Infancy: Sensory motor period } \\
\text { At its end, evidence of an internal } \\
\text { representational system. } \\
\text { Symbolizing the } \\
\text { problem-solving sequence } \\
\text { before actually responding. } \\
\text { Deferred imitation. }\end{array}$ \\
\hline $2-4 y$ & $\begin{array}{l}\text { Childhood: Preoperational period } \\
\text { Increased use of verbal } \\
\text { representation, but speech is } \\
\text { egocentric. The beginnings of } \\
\text { symbolic rather than simple } \\
\text { motor play. Transductive } \\
\text { reasoning. Can think about } \\
\text { something without the object } \\
\text { being present by use of } \\
\text { language. }\end{array}$ \\
\hline
\end{tabular}

and weaning (while continuing to depend on older people for food), and on behavioral characteristics, including immature motor control. The evolutionary advantage of childhood lay in the mother's freedom to discontinue breastfeeding her 3-y-old infant to initiate a new pregnancy. This enhancement of reproductive output and the social interaction of an extended family and extra-familial ties do not put the mother, her infant, or her older children at risk. As a consequence of the childhood stage, more humans survive to adulthood than any other mammal.

Piaget defined childhood as the preoperational stage-from age 2, when he starts to talk, to age 4 (Table 1) (6). Applying his new knowledge of language, the child begins to use symbols to represent objects. Early in this stage, he also personifies objects. He is now better able to think about things and events that are not immediately present. Oriented to the present, the child has difficulty conceptualizing time. His thinking is influenced by fantasy - the way he would like things to be-and he assumes that others see situations from his viewpoint. He takes in information and then changes it in his mind to fit his ideas.

\section{INFANCY-CHILDHOOD GROWTH TRANSITION}

Based on the analysis of growth parameters, the Infancy, Childhood, and Puberty (ICP) growth model divided human growth into three successive and partly superimposed phases that reflect the endocrine control mechanisms of the growth process (7) (Fig. 1). The infancy phase of the ICP model has been assumed to begin at mid-gestation and to tail off at approximately 2-3 y of age, representing the postnatal extension of fetal growth, and is regarded as being nutrition dependent and closely linked to the action of insulin-like growth factors (IGFs) $(8,9)$.

The childhood growth phase starts in affluent Western countries between 6 and 12 mo of age $(7,10)$, and continues through puberty until growth ceases. Thus, the ICP model proposes a period of transition, whereby the initiation of the childhood growth phase overlaps with the infancy growth phase and the infantile life history stage, as defined by weaning at 2-3 y of age.

After gradual deceleration of postnatal infantile growth, the growth rate abruptly increases between 6 and 12 mo of age. This defines the onset of the childhood growth transition (IC-transition; Fig. 1) $(7,11)$.

The negative impact of a DICT on attained height is illustrated in Figures 1 and 2. It has been shown that each month of delay results in a growth deficit of $0.4 \mathrm{~cm}$ in boys and $0.5 \mathrm{~cm}$ in girls at age $5 \mathrm{y}(12)$. Hence, an early or late IC growth transition within the normal range of 6-12 mo accounts for a difference of 2.4 and $3.0 \mathrm{~cm}$ in boys and girls, respectively, and a severely delayed IC growth transition to as late as 18 mo produces a deficit of 4.8 and 6.0 $\mathrm{cm}$, in boys and girls, respectively, at age 5, compared with individuals who have an early onset of the IC growth transition. The change in height standard deviation score from age 0.5 to 18 y has been calculated to be $1.23-0.09 \times$ age at IC growth transition (Fig. 2). 


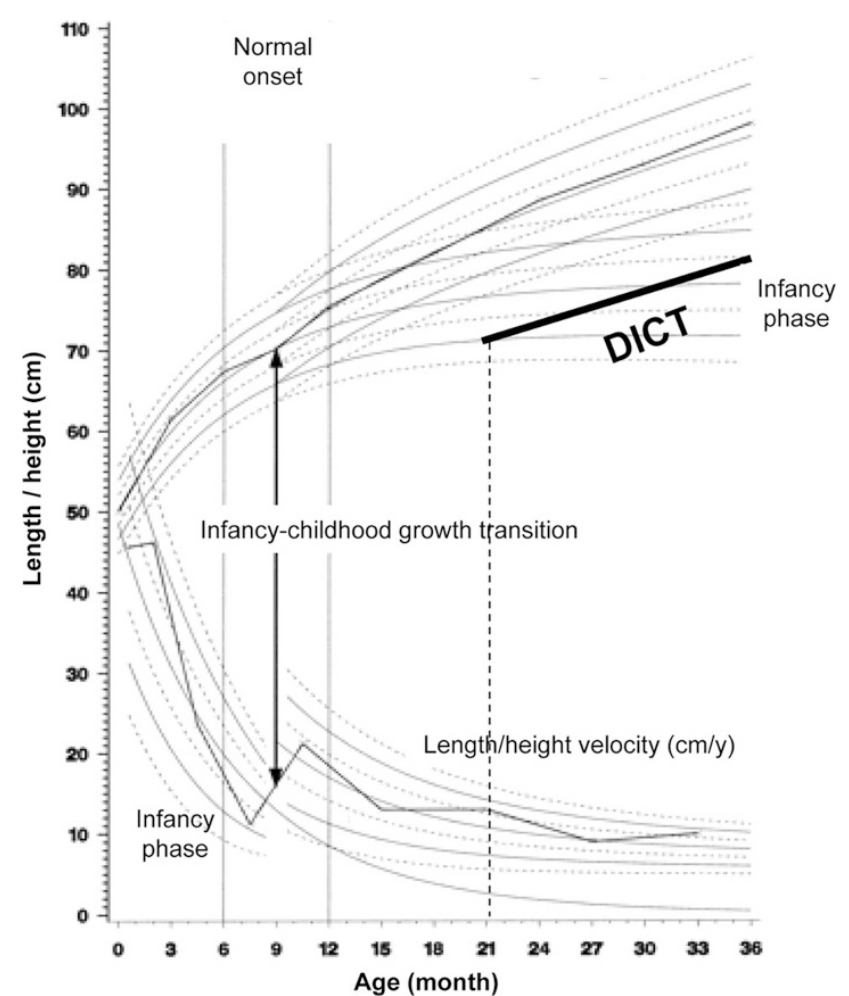

Figure 1. The Infancy, Childhood, and Puberty (ICP) growth model divides human growth into three successive and partly superimposed phases that reflect the control mechanisms of the growth process. The infancy phase of the ICP model begins at mid-gestation and tails off at $2-3$ y of age. The childhood growth phase sets on in affluent Western countries between 6 and 12 mo of age, and when the transition is delayed (DICT) beyond age 12 mo (21 mo for this hypothetical child), it has a permanent effect on final adult height. Figure adapted from Xu et al., Pediatr Res 51:377-385 Copyright () 2002 The International Pediatric Research Foundation, with permission.

Children with DICT have normal infantile and normal childhood growth patterns, indicating normally functioning growth control mechanisms, and the only abnormal event is their delayed IC growth transition $(13,14)$. This is observed in as many as $44 \%$ of children with ISS or small for gestational age (SGA) (Fig. 3) (Kristrom et al., Delayed infancychildhood spurt (DICS) in idiopathic short stature. 46th Annual Meeting of the European Society for Pediatric Endocrinology, September 27-30, 2007, Helsinki, Finland, Abstract PO3-538; Zuckerman-Levin I et al., Delayed infancychildhood spurt in SGA children with no catch up growth. 46th Annual Meeting of the European Society for Pediatric Endocrinology, September 27-30, 2007, Helsinki, Finland, Abstract PO3-537). This effect is time dependent, with a longer delay resulting in shorter prepubertal stature (15).

\section{ENDOCRINE CONTROL OF IC GROWTH TRANSITION}

The IC growth transition represents the age at which growth hormone $(\mathrm{GH})$ begins to regulate growth significantly, and reflects the control of growth by the GH-IGF-I endocrine axis and target cell responsiveness $(16,17)$. The IC growth transition occurs in parallel with a rise in serum levels of GHdependent IGF-I and IGF-binding protein-3 during the second

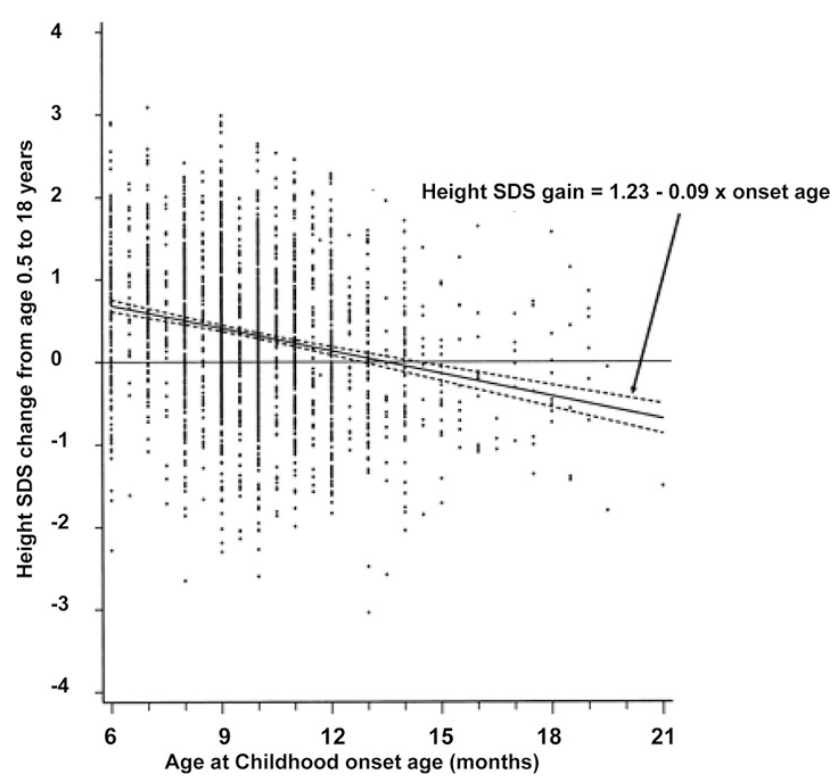

Figure 2. The negative impact on height gain from 0.5 to $18 \mathrm{y}$ of age by a delay in IC transition (DICT). Figure adapted from Liu Y et al., Pediatr Res 47:475-480 Copyright (C) 2000 The International Pediatric Research Foundation, with permission.

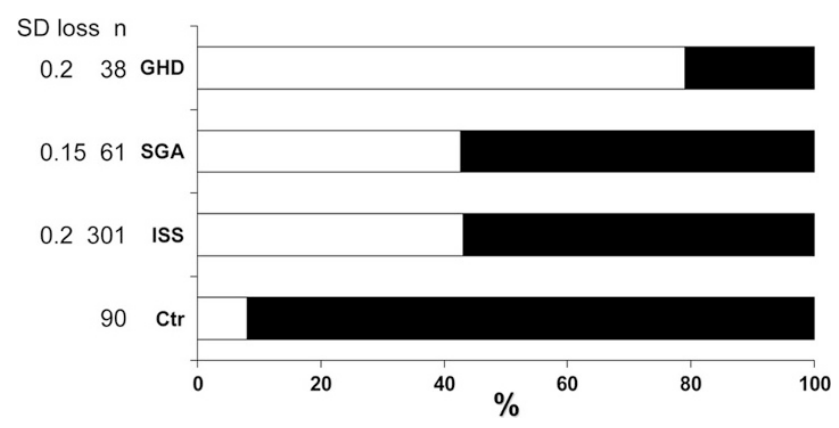

Figure 3. Percentage of children affected by DICT among children with ISS, short SGA and GHD compared with Israeli controls. The left columns give the impact of each month of delay on height loss in SDS for each group.

half of the first year of life, and children with a delayed IC growth transition show a delay in the 6-12 mo rise of IGF-I levels (Fig. 4) $(8,9)$. Moreover, an IC growth transition is absent in children with GH deficiency who receive no hormonal therapy (16).

\section{RESPONSE TO ENERGY CRISIS}

Major trends in human evolution were precipitated by large-scale ecosystem changes, and research on bioenergetics has become a central component of ecosystem ecology. These disciplines now examine how individuals and populations extract energy from their environment, and how, in turn, that energy is allocated for biologic processes such as maintenance, activity, growth, and reproduction. Life history tradeoffs result from limitations in the availability of critical resources such as energy or nutrients, necessitating decisions on the differential allocation of resources to costly traits. Diet is directly related to energy harvest, and body size is also firmly intertwined with life history because it is critical to energy 

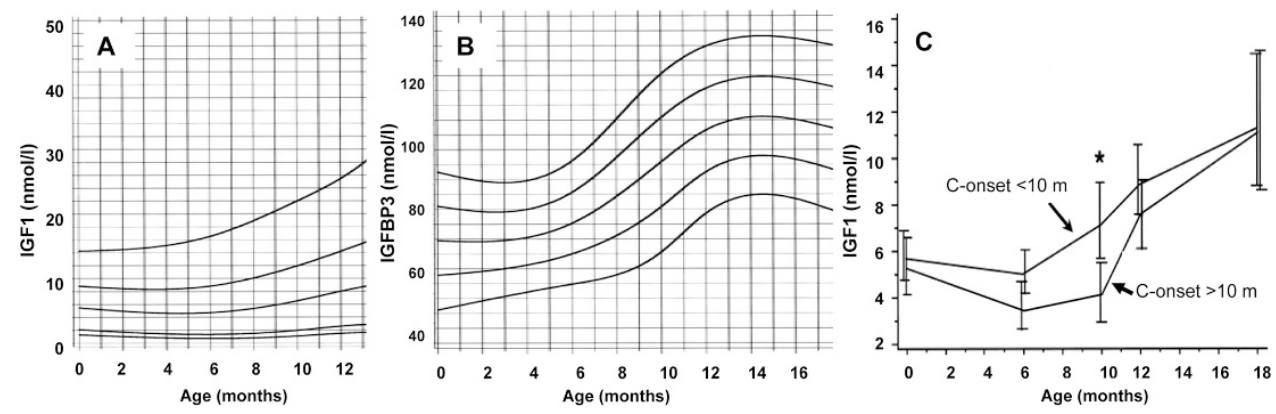

Figure 4. The IC growth transition occurs in parallel with a rise in serum levels of GH-dependent IGF-I $(A)$ and IGF-binding protein-3 $(B)$ during the second half of the first year of life, and children with a delayed IC growth transition $(C)$ show a delay in the 6 to 12 mo rise of IGF-I levels. Figure adapted from Low LC et al. Pediatr Res 50:737-742 Copyright (C) 2001 The International Pediatric Research Foundation, with permission. budget. At any given body size, a species that extracts higher quality foods from the environment has increased life history options for evolutionary fitness. On the other hand, while requiring more total energy, increasing body size improves heat retention and lessens the relative energy needed per gram of tissue (18). An obvious tradeoff for the metabolic cost, large body size decreases mortality, because there are always fewer predators for large mammals than for small ones.

The reasons for the hominid's evolving bigger size are not quite clear, but most probably were related to the changing environment, which provided access to richer foods and favored stronger bodies for big-game hunting. About 30,000 y ago, hunter-gatherer Cro-Magnon humans reached a peak male height of $174-178 \mathrm{~cm}$ (men being the same 10-15 cm taller than females as they are today). With the subsequent Ice Age and scarcer big game, many populations switched to farming and lesser food quality, while great body strength was not necessarily at a premium any more. Farmers became shorter, and then much shorter when they urbanized and industrialized. In about 1800 , heights took a dip as industrial towns became polluted and overcrowded. Although this has been a process of evolutionary biology in its gradual cumulative selection by small steps of genetic adaptation to the environment (19), the gradual secular trend in growth and puberty since about 1840 (20) can hardly be imagined to result from gene base sequence modifications. The world trend represents the impact of environmental forces applied to an inherent adaptive plasticity.

For the IC growth transition to occur, the child must have a positive energy balance. The age when the IC growth transition occurs is influenced and delayed by disease (21), when energy consumption increases rapidly, and by undernutrition, gastrointestinal infection, and socioeconomic impediments $(12,15)$, with insufficient energy supplies.

A delayed IC growth transition is the main mechanism resulting in short stature in children living in poor areas of developing countries. In a community-based longitudinal study in Lahore, Pakistan, the median ages of the IC growth transitions were at 15, 13, 10, and 9 mo in the suburban, village, urban, and Swedish control groups, respectively (15). Among the poorest suburban children of Lahore, who suffer frequent infections and undernutrition, 35\% have DICT, and among the poor children of Malawi, Africa, as many as $60 \%$ have a delayed IC growth transition (15). Many of them have an IC growth transition that occurs at 3-4 y of age, which compromises their prepubertal height by $15-20 \mathrm{~cm}$, culminat- ing in mean adult male and female heights of 162.5 and 155 $\mathrm{cm}$, respectively (22).

\section{THE IC GROWTH TRANSITION AS AN EVOLUTIONARY TACTIC}

Charles Darwin observed that: “... we do not always bear in mind, that though food may be now superabundant, it is not so at all seasons of each recurring year ... A large number of eggs is of some importance to those species, which depend on a rapidly fluctuating amount of food, for it allows them rapidly to increase in number." (23). This is probably the first suggestion for plasticity (phenotypic changeability) in adjusting to changing environments.

Unlike short periods of stunting at any other age, a delayed IC growth transition is not followed by catchup growth. In our analysis of 176 growth charts of DICT children, we did not encounter a single case of catchup growth. We propose that the long transition period from IC growth phases corresponds to a period of plasticity in growth that has evolved to adjust an individual's growth to environmental circumstances, such as limited energy resources. The ability of the genotype to produce different phenotypes in response to environmental circumstances, and plasticity (the ability of the individual to modify phenotypic response to altered environmental conditions) of the timing of life history transitions, such as the IC growth transition, is evolutionary strategy and the basis for this adaptability (24).

In the evolutionary timescale, energy crises persist beyond a single generation, and, indeed, the timing of the IC growth transition has been shown to be inherited. A negative correlation was demonstrated between the age of the IC growth transition and mid-parental height, indicating that offspring of ancestors, who might have responded to energy crises in their infancy, have a relative delay in the IC growth transition (25).

A great deal of attention has been paid to intrauterine nutritional "fetal programming" leading to a "thrifty phenotype" and later health consequences, as recently reviewed (26). In terms of fetal programming of final height, the vast majority of infants with intrauterine growth retardation catch up immediately after birth and end up with a normal stature. Hence, in evolutionary terms, fetal growth, which depends on uterine and placental function, is too labile a phase to affect final height. On the other hand, a delay in the IC growth transition is not associated with catch-up growth, indicating that events during the first year of life affect ultimate height. In 
analyzing close to 400 children with ISS or SGA, we have not encountered any single case who showed a catchup growth.

Although the evolutionary origin of the switch and control mechanisms for the onset of the childhood growth phase is genetic, we speculate that the plasticity of the IC growth transition switch may be epigenetic, causing heritable changes in gene function without changing the DNA sequence. We concede the lack of any experimental epigenetic studies to support this speculation. Epigenetic changes occurring at certain stages of development become incorporated into the genome, providing transgenerational passage of environmentally induced epigenetic changes (27), and endocrine, physiologic, and behavioral traits persist even after the original selection pressure is relaxed (28) for at least three generations (29). Because such events can occur at a rate up to 100,000 times higher than point mutations, epigenetic changes potentially have a much greater impact on morphologic adaptive evolution (30).

In the case of a delayed IC growth transition, hormones, cytokines, and nutrition may account for epigenomic evolutionary adaptations to energy crises. Hormones have been shown to epigenetically imprint genes, and hormone target genes are liable to be epigenetically modified. Hormonal exposure early in life alters the response to hormonally regulated genes to the same or different hormones later in life; the first hormone experience alters the set point epigenetically for a later hormone response. More specifically, exposure to estrogens early in life compromise health later in life, fetal glucocorticoids imprint later glucocorticoid responses (31), demethylation of the leptin promoter correlates with preadipocyte differentiation into adipocytes (32), and leptin has a trophic effect on plasticity of hypothalamic responses to nutrients that regulate feeding (33). As a delay in the IC growth transition is associated with delayed development of the GHIGF-I axis, it is yet to be defined whether such changes occur at the level of hormone secretion or target cell response.

Nutritional deprivation during infancy, even if transient, may have a lasting influence on the expression of genes by interacting with epigenetic mechanisms and altering chromatic conformation and transcription factor accessibility (34). Moreover, epigenetic programming by energy crises has been shown to be transmitted to the next generation (35).

\section{DELAYED GROWTH TRANSITION, SHORT STATURE, AND OBESITY}

A delay in the IC growth transition is an adaptive strategy to an energy crisis during infancy. Yet, evolutionary adaptation occurs at a time scale, which assumes that the same environmental conditions will prevail. It does not take into account the energy saturation of modern society. Indeed, the transition from IC stages of the human life history is also the timing of shift in modern dietary energy saturation in affluent societies, and as a result, evolutionary adaptation to an apparent energy crisis results in the eventual unwanted permutation of obesity with short stature (36). Indeed, stunted children (44\% of which have DICT) show higher susceptibility to the effects of a high-fat diet (37).

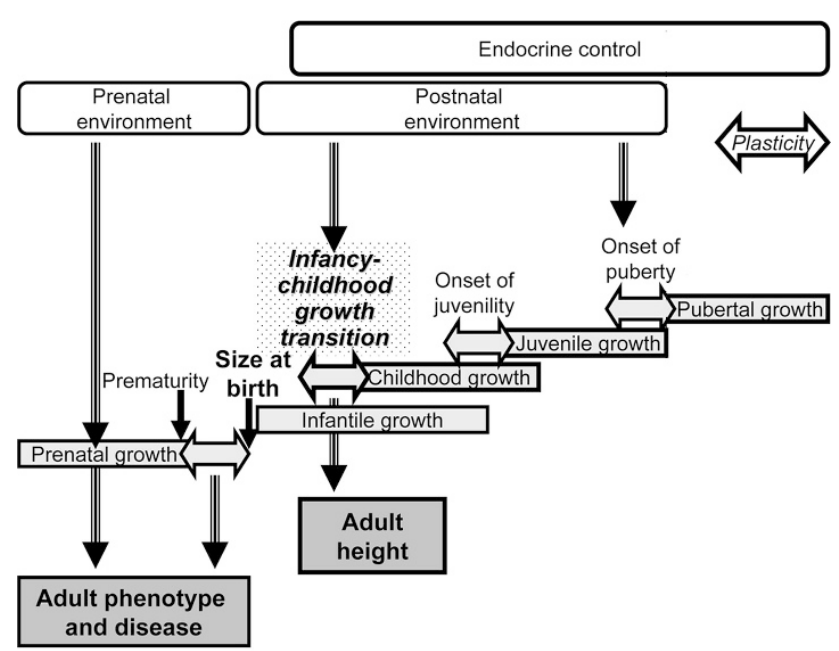

Figure 5. Life history stages and their transition to one another respond to environmental cues. The transition from infancy to childhood is the most important determinant of final adult height.

\section{CONCLUSIONS}

A delay in the onset of childhood growth phase provides an evolutionary adaptive mechanism in the event of an energy crisis, culminating in shortness of stature that is appropriate for energy shortage but unwanted in energy-saturated affluent societies (Fig. 5). Clinical diagnosis of this childhood growth delay requires a careful analysis of infantile growth data, and the DICT accounts for a significant proportion of what has been termed ISS.

Although many questions remain to be answered, the concept of a delay in the childhood growth transition, with its evo-devo implications, provides a new direction for research into child growth.

\section{REFERENCES}

1. Muller GB 2007 Evo-devo: extending the evolutionary synthesis. Nat Rev Genet 8:943-949

2. Hochberg Z 2008 Juvenility in the context of life history theory. Arch Dis Child [Epub ahead of print]

3. Bogin B 1999 Evolutionary perspective on human growth. Annu Rev Anthropol 28:109-153

4. Bogin B 2002 The evolution of human growth. In: Cameron N (ed) Human Growth and Development. Academic Press, Amsterdam, pp 295-320

5. Washburn SL 1960 Tools and human evolution. Sci Am 203:63-75

6. Singer D, Revenson, TA 1998 A Piaget Primer; How a Child Thinks. International Universities Press, Inc, Madison

7. Karlberg J, Engstrom I, Karlberg P, Fryer JG 1987 Analysis of linear growth using a mathematical model. I. From birth to three years. Acta Paediatr Scand 76:478-488

8. Wang HS, Chard T 1992 The role of insulin-like growth factor-I and insulin-like growth factor-binding protein-1 in the control of human fetal growth. J Endocrinol 132:11-19

9. Leger J, Oury JF, Noel M, Baron S, Benali K, Blot P, Czernichow P 1996 Growth factors and intrauterine growth retardation. I. Serum growth hormone, insulin-like growth factor (IGF)-I, IGF-II, and IGF binding protein 3 levels in normally grown and growth-retarded human fetuses during the second half of gestation. Pediatr Res 40:94-100

10. Low LCK, Tam SYM, Kwan EYW, Tsang AMC, Karlberg J 2001 Onset of significant GH dependence of serum IGF-I and IGF-binding protein 3 concentrations in early life. Pediatr Res 50(6):737-742

11. Karlberg J 1987 On the modelling of human growth. Stat Med 6:185-192

12. Liu Y, Albertsson-Wikland K, Karlberg J 2000 Long-term consequences of early linear growth retardation (stunting) in Swedish children. Pediatr Res 47:475-480

13. Van den Broeck J, Brand R, Massa G, Herngreen WP, Wit JM 2000 Length velocity acceleration at 9 months of age in a representative birth cohort of Dutch infants. J Pediatr Endocrinol Metab 13:45-54

14. Rekers-Mombarg LT, Cole TJ, Massa GG, Wit JM 1997 Longitudinal analysis of growth in children with idiopathic short stature. Ann Hum Biol 24:569-583 
15. Liu YX, Jalil F, Karlberg J 1998 Growth stunting in early life in relation to the onset of the childhood component of growth. J Pediatr Endocrinol Metab 11:247-260

16. Karlberg J, Albertsson-Wikland K 1988 Infancy growth pattern related to growth hormone deficiency. Acta Paediatr Scand 77:385-391

17. Wit JM, van Unen H 1992 Growth of infants with neonatal growth hormone deficiency. Arch Dis Child 67:920-924

18. Schmidt-Nielsen K 1975 Scaling in biology: the consequences of size. J Exp Zool 194:287-307

19. Roff DA 2007 Contributions of genomics to life-history theory. Nat Rev Genet 8:116-125

20. Arcaleni E 2006 Secular trend and regional differences in the stature of Italians, 1854-1980. Econ Hum Biol 4:24-38

21. Karlberg J, Jalil F, Lam B, Low L, Yeung CY 1994 Linear growth retardation in relation to the three phases of growth. Eur J Clin Nutr 48(suppl 1):S25-S43; discussion S43-S24

22. Zverev Y, Chisi J 2004 Anthropometric indices in rural Malawians aged 45-75 years. Ann Hum Biol 31:29-37

23. Darwin C 1859 The struggle for existance. In: On the Origin of Species by Means of Natural Selection or The Preservation of Favoured Races in the Struggle for Life. John Murray, London, pp 60-79

24. Kuzawa CW 2005 Fetal origins of developmental plasticity: are fetal cues reliable predictors of future nutritional environments? Am J Hum Biol 17:5-21

25. Xu X, Wang W, Guo Z, Karlberg J 2002 Longitudinal growth during infancy and childhood in children from shanghai: predictors and consequences of the age at onset of the childhood phase of growth. Pediatr Res 51:377-385

26. Symonds ME, Gardner DS 2006 Experimental evidence for early nutritional programming of later health in animals. Curr Opin Clin Nutr Metab Care 9:278-283
27. Anway MD, Cupp AS, Uzumcu M, Skinner MK 2005 Epigenetic transgenerational actions of endocrine disruptors and male fertility. Science 308:1466-1469

28. Sollars V, Lu X, Xiao L, Wang X, Garfinkel MD, Ruden DM 2003 Evidence for an epigenetic mechanism by which Hsp90 acts as a capacitor for morphological evolution. Nat Genet 33:70-74

29. Campbell JH, Perkins P 1988 Transgenerational effects of drug and hormonal treatments in mammals: a review of observations and ideas. Prog Brain Res 73:535-553

30. Ellegren H 2000 Microsatellite mutations in the germline: implications for evolutionary inference. Trends Genet 16:551-558

31. Moritz KM, Boon WM, Wintour EM 2005 Glucocorticoid programming of adult disease. Cell Tissue Res 322:81-88

32. Melzner I, Scott V, Dorsch K, Fischer P, Wabitsch M, Bruderlein S, Hasel C, Moller P 2002 Leptin gene expression in human preadipocytes is switched on by maturation-induced demethylation of distinct $\mathrm{CpGs}$ in its proximal promoter. J Biol Chem 277:45420-45427

33. Bouret SG, Draper SJ, Simerly RB 2004 Trophic action of leptin on hypothalamic neurons that regulate feeding. Science 304:108-110

34. Waterland RA, Garza C 1999 Potential mechanisms of metabolic imprinting that lead to chronic disease. Am J Clin Nutr 69:179-197

35. Waterland RA, Jirtle RL 2004 Early nutrition, epigenetic changes at transposons and imprinted genes, and enhanced susceptibility to adult chronic diseases. Nutrition 20:63-68

36. Hermanussen M, Garcia AP, Sunder M, Voigt M, Salazar V, Tresguerres JA 2006 Obesity, voracity, and short stature: the impact of glutamate on the regulation of appetite. Eur J Clin Nutr 60:25-31

37. Sawaya AL, Martins PA, Grillo LP, Florencio TT 2004 Long-term effects of early malnutrition on body weight regulation. Nutr Rev 62:S127-S133 